\title{
ASYMPTOTICS OF SHARP CONSTANTS OF MARKOV-BERNSTEIN INEQUALITIES IN INTEGRAL NORM WITH JACOBI WEIGHT
}

\author{
A. I. APTEKAREV, A. DRAUX, V. A. KALYAGIN, AND D. N. TULYAKOV
}

(Communicated by W. Van Assche)

\begin{abstract}
The classical A. Markov inequality establishes a relation between the maximum modulus or the $L^{\infty}([-1,1])$ norm of a polynomial $Q_{n}$ and of its derivative: $\left\|Q_{n}^{\prime}\right\| \leqslant M_{n} n^{2}\left\|Q_{n}\right\|$, where the constant $M_{n}=1$ is sharp. The limiting behavior of the sharp constants $M_{n}$ for this inequality, considered in the space $L^{2}\left([-1,1], w^{(\alpha, \beta)}\right)$ with respect to the classical Jacobi weight $w^{(\alpha, \beta)}(x):=(1-x)^{\alpha}(x+1)^{\beta}$, is studied. We prove that, under the condition $|\alpha-\beta|<4$, the limit is $\lim _{n \rightarrow \infty} M_{n}=1 /\left(2 j_{\nu}\right)$ where $j_{\nu}$ is the smallest zero of the Bessel function $J_{\nu}(x)$ and $2 \nu=\min (\alpha, \beta)-1$.
\end{abstract}

\section{INTRODUCTION}

A quantity

$$
M_{n}:=\sup _{\operatorname{deg} Q_{n} \leqslant n} \frac{\left\|Q_{n}^{\prime}\right\|_{\mathfrak{X}_{1}}}{\left\|Q_{n}\right\|_{\mathfrak{X}_{2}}}, \quad Q_{n} \text { - polynomial, }
$$

is called the sharp constant for the Markov-Bernstein inequality in functional spaces $\mathfrak{X}_{1}, \mathfrak{X}_{2}$ with norms $\|\cdot\|_{\mathfrak{X}_{1}},\|\cdot\|_{\mathfrak{X}_{2}}$.

The remarkable classical inequality of A.A. Markov for $\mathfrak{X}_{1}=\mathfrak{X}_{2}=L^{\infty}([-1,1])$

$$
\left\|Q_{n}^{\prime}\right\| \leqslant n^{2}\left\|Q_{n}\right\|
$$

is sharp [1. We recall that the corresponding inequality for the trigonometric polynomials was first obtained by S.N. Bernshtein in [2]. His result was not sharp, and the sharp version is due to E. Landau (see [3]). For the weighted $L^{2}$ spaces $\mathfrak{X}_{1}=\mathfrak{X}_{2}:=L^{2}([a, b], w)$, for some classical weights, the sharp constants (1.1) are known (see [4, pp. 570-571]):
1. $w=\exp \left(-x^{2}\right), x \in(-\infty, \infty), M_{n}=\sqrt{2 n}$
2. $w=\exp (-x), \quad x \in(0, \infty), \quad M_{n}=\frac{1}{2 \sin \left(\frac{\pi}{4 n+2}\right)}$
(E. Schmidt, 1944),
(P. Turán, 1960).

However, for other classical weights, explicit expressions for the sharp constants are not known. In [8] results on the asymptotics $M_{n} \rightarrow$ ?,$n \rightarrow \infty$ were discussed.

Received by the editors June 11, 2013 and, in revised form, February 24, 2014 and April 18, 2014 .

2010 Mathematics Subject Classification. Primary 41A20.21, 33C45, 42C05; Secondary 47B99, $30 \mathrm{~B} 70$.

The first and fourth authors were partly supported by the program N1 of DMS RAS and grants RFBR-13-01-12430, RFBR-14-01-00604. The third author was partly supported by the Scientific Schools program - 2900.2014.1. The paper was finished while the first author visited INSA, Rouen, France. 
In particular, for the Gegenbauer weight $w^{(\alpha)}(x):=\left(1-x^{2}\right)^{\alpha}, x \in[-1,1], \alpha>-1$, the following result was stated there

$$
M_{n}=\frac{n^{2}}{2 j_{\nu(\alpha)}}(1+o(1)), \quad \nu(\alpha):=\frac{\alpha-1}{2},
$$

where $j_{\nu}$ is the smallest zero of the Bessel function $J_{\nu}(x)$ (we shall keep the notation $\nu(\alpha)$ and $j_{\nu}$ in what follows).

In the present paper we study the asymptotics of the sharp constant (1.1) for the classical Jacobi weight (the space $L^{2}\left([-1,1], w^{(\alpha, \beta)}\right)$ as defined in Section 2.1):

$$
\mathfrak{X}_{1}=\mathfrak{X}_{2}:=L^{2}\left([-1,1], w^{(\alpha, \beta)}\right), w^{(\alpha, \beta)}(x):=(1-x)^{\alpha}(x+1)^{\beta}, \alpha, \beta>-1 .
$$

The main result of our paper is

Theorem 1.1. Let the parameters of the Jacobi weight (1.3) satisfy the restriction

$$
w^{(\alpha, \beta)}(x): \quad|\alpha-\beta|<4 .
$$

Then, for the sharp constant (1.1) in the space (1.3), we have the asymptotics:

$$
M_{n}=\frac{n^{2}}{2 j_{\nu^{*}}}(1+o(1)), \quad \nu^{*}=\min \{\nu(\alpha), \nu(\beta)\} .
$$

We see that, for $\alpha=\beta$, asymptotics (1.5) match (1.2). When $\alpha \neq \beta$, then asymptotics (1.5) appear as a reasonable generalization of (1.2). The most surprising for us is the appearance of the restriction (1.4). At the moment we cannot prove or disapprove its necessity, however, we have to admit that this restriction is unavoidable in our proof strategy of Theorem 1.1 .

The rest of the paper contains the proof of Theorem 1.1. Our approach consists of the following steps:

1) We start with an explicit representation of $M_{n}$ as the eigenvalue of a linear operator in $\mathbb{R}^{n}$ defined by a five diagonal matrix.

2) Then we state a Finite Difference (FD) Boundary Value Problem (BVP) which is equivalent to the eigenvalue problem.

3) The next step is to determine a limiting (for FD problem) Differential Equation (DE) and its general solution.

4) Then we vanish the spectral parameter in the FD problem and find linearly independent Particular Solutions (PS) satisfying Boundary Conditions (BC) at the initial values of the discrete variable (the left end $\mathrm{BC}$ ). For the small (with respect to $n$ ) indexes in FD and spectral parameter in the fixed range, the asymptotics of the solutions of FD does not depend on the spectral parameter. Therefore, the initial conditions can be rewritten as asymptotics conditions for the indices $1<<k<<n$. Then this condition is exported to the boundary condition of DE.

5) Matching these FD problems, we get PS of the limiting DE.

6) Finally, taking these PS of DE as an approximation of the PS of FD, we satisfy the right end BC of FD BVP. It gives an approximation of the desired eigenvalue as in (1.2) or (1.5).

These steps are performed in Section 2. Some of these steps have already been studied before for various functional spaces in (1.1), see in 4, Chapter 6 Section 6.1.6], 9], 8, [10, [11. 
However, to conclude a rigorous proof of Theorem 1.1, it remains to justify the final step, i.e. to prove that PS of DE which match the satisfying to the left end $\mathrm{BC}$ of FD problem, indeed are close to the PS of FD problem. In Section 3 we state and prove the corresponding result, see Theorem 3.1. This theorem establishes a new result on the local asymptotics of the powerlike growing solution of the high order recurrence relations. Previous results in this direction are in [12], 14, [13. In all of the following we will use the factorial notation $(a)$ ! for the value of $\Gamma(a+1)$.

\section{Finite diffERence BVP for $M_{n}$ AND its Differential approximation}

2.1. A spectral representation for $M_{n}$ in $\mathbb{R}^{n}$. We note from (1.1), that $M_{n}$ is the norm of the operator differentiation in a finite dimensional space $\mathcal{P}_{n}$ of polynomials of degree at most equal to $n$. Let $Q_{n}$ be an arbitrary polynomial of $\mathcal{P}_{n}$. We take the expansion of this polynomial $Q_{n}$ and of its derivative $Q_{n}^{\prime}$ in the basis of monic Jacobi polynomials $P_{k}^{(\alpha, \beta)}\left(P_{k}^{(\alpha, \beta)}(x)=x^{k}+\cdots\right)$. By using the $L^{2}\left([-1,1], w^{(\alpha, \beta)}\right)$ inner product

$$
(g, f):=\int_{-1}^{1} g(x) \overline{f(x)} w^{(\alpha, \beta)}(x) d x
$$

the square norm of $P_{n}^{(\alpha, \beta)}$ is

$$
\left\|P_{n}^{(\alpha, \beta)}\right\|^{2}=\left(P_{n}^{(\alpha, \beta)}, P_{n}^{(\alpha, \beta)}\right)=2^{2 n+\alpha+\beta-1} \frac{n !(n+\alpha) !(n+\beta) !(n+\alpha+\beta) !}{(2 n+\alpha+\beta) !(2 n+\alpha+\beta+1) !} .
$$

Then, we have (in general $Q_{n}=\sum_{k=0}^{n} c_{k} P_{k}^{(\alpha, \beta)}$ but to solve (1.1), that it is sufficient to consider the case where $\left.c_{0}=0\right)$ :

$$
Q_{n}^{\prime}(x):=\sum_{k=0}^{n-1} v_{k} P_{k}^{(\alpha, \beta)}, \quad Q_{n}(x):=\sum_{k=0}^{n-1} u_{k} P_{k+1}^{(\alpha, \beta)} .
$$

Differentiating $Q_{n}$ here, and using the property of Jacobi polynomials

$$
\frac{d}{d x} P_{k}^{(\alpha, \beta)}(x)=k P_{k-1}^{(\alpha+1, \beta+1)}(x),
$$

we arrive at

$$
Q_{n}^{\prime}=\sum_{k=0}^{n-1} u_{k} P_{k+1}^{\prime(\alpha, \beta)}=\sum_{k=0}^{n-1}(k+1) u_{k} P_{k}^{(\alpha+1, \beta+1)}=\sum_{k=0}^{n-1} v_{k} P_{k}^{(\alpha, \beta)} .
$$

Then, applying the well-known $\alpha$ increasing (and $\beta$ increasing) relation (see [5, Chapter 22]):

$$
P_{n}^{(\alpha, \beta)}=P_{n}^{(\alpha+1, \beta)}-\frac{2 n(n+\beta) P_{n-1}^{(\alpha+1, \beta)}}{(2 n+\alpha+\beta)(2 n+\alpha+\beta+1)},
$$

we obtain for the vectors from (2.3)

$$
\vec{v}:=\left(v_{0}, v_{1}, \ldots, v_{n-1}\right)^{T r}, \quad \vec{u}:=\left(u_{0}, u_{1}, \ldots, u_{n-1}\right)^{T r},
$$

the following relations

$$
\mathbf{N} \vec{u}=\mathbf{C}_{2} \mathbf{C}_{1} \vec{v}, \quad \mathbf{N}:=\operatorname{diag}(1,2, \ldots, n),
$$


and for the $n \times n$ matrices $\mathbf{C}_{2}, \mathbf{C}_{1}$ we have from (2.4)

$$
\begin{aligned}
& \mathbf{C}_{1}:=\mathbf{I}-\operatorname{diag}\left(\frac{2 k(k+\beta)}{(2 k+\alpha+\beta)(2 k+\alpha+\beta+1)}\right)_{k=1}^{n} \mathbf{T} \\
& \mathbf{C}_{2}:=\mathbf{I}+\operatorname{diag}\left(\frac{2 k(k+\alpha+1)}{(2 k+\alpha+\beta+1)(2 k+\alpha+\beta+2)}\right)_{k=1}^{n} \mathbf{T}
\end{aligned}, \quad \mathbf{T}:=\left(\begin{array}{ccccc}
0 & 1 & & & 0 \\
& 0 & 1 & & \\
& & 0 & \ddots & \\
& & & \ddots & 1 \\
0 & & & & 0 \\
0 & 0 & & & 0
\end{array}\right) .
$$

Now, we write norms for (2.3) by using the inner product (2.1):

$$
\begin{gathered}
\left\|Q_{n}^{\prime}\right\|^{2}=<\vec{v}, \mathbf{D} \vec{v}>, \quad \mathbf{D}:=\operatorname{diag}\left(\left\|P_{k}^{(\alpha, \beta)}\right\|^{2}\right)_{k=0}^{n-1}, \\
\left\|Q_{n}\right\|^{2}=<\vec{u}, \mathbf{D}^{+} \vec{u}>, \quad \mathbf{D}^{+}:=\operatorname{diag}\left(\left\|P_{k}^{(\alpha, \beta)}\right\|^{2}\right)_{k=1}^{n},
\end{gathered}
$$

where $<,>$ is the usual vector inner product. Thus, for the sharp constant in (1.1) - (1.3) we have by using (2.5)

$$
M_{n}^{2}=\sup _{\vec{v}} \frac{<\vec{v}, \mathbf{D} \vec{v}\rangle}{\left\langle\mathbf{N}^{-1} \mathbf{C}_{2} \mathbf{C}_{1} \vec{v}, \mathbf{D}^{+} \mathbf{N}^{-1} \mathbf{C}_{2} \mathbf{C}_{1} \vec{v}\right\rangle}=\sup _{\vec{v}} \frac{\langle\vec{v}, \mathbf{D} \vec{v}\rangle}{\langle\vec{v}, \mathbf{A} \vec{v}\rangle},
$$

where we denote

$$
\mathbf{A}:=\mathbf{C}_{1}^{T r} \mathbf{C}_{2}^{T r} \mathbf{N}^{-1} \mathbf{D}^{+} \mathbf{N}^{-1} \mathbf{C}_{2} \mathbf{C}_{1}, \mathbf{D}:=\operatorname{diag}\left(d_{k}\right)_{k=0}^{n-1}, \mathbf{D}^{+}:=\operatorname{diag}\left(d_{k}\right)_{k=1}^{n} .
$$

For the purpose of (2.6) we can omit the factor $2^{\alpha+\beta-1}$ in (2.2), so we put

$$
d_{k}:=\frac{2^{2 k} k !(k+\alpha) !(k+\beta) !(k+\alpha+\beta) !}{(2 k+\alpha+\beta) !(2 k+\alpha+\beta+1) !} .
$$

Finally, from (2.6) we get by the arguments of a pencil of quadratic forms (see [6. Chapter 10.7]) the spectral radius representation for the exact constant:

$$
M_{n}^{2}=\lambda_{\min }^{-1}(\mathbf{A}, \mathbf{D}),
$$

where $\lambda_{\min }(\mathbf{A}, \mathbf{D})$ is a root (with the minimal modulus) of the equation

$$
\operatorname{det}(\mathbf{A}-\lambda \mathbf{D})=0,
$$

and correspondingly the eigenvector $\vec{v}$

$$
\left(\mathbf{A}-\lambda_{\min } \mathbf{D}\right) \vec{v}=0
$$

defines the extremal polynomial (2.3) in (1.1), (1.3).

2.2. Finite difference equation for the coordinates of $\vec{v}$. To simplify expressions (i.e. to cancel factorials) in what follows, we introduce a new variable for the coordinates of the vector $\vec{v}$ (see (2.3):

$$
v_{k}=: x_{k} \frac{(2 k+\alpha+\beta+1) !}{2^{k}(k+\alpha) !(k+\beta) !}, \quad k=0, \ldots, n-1 .
$$

Next, taking the $k$-th coordinate of the equation (2.11)

$$
[(\mathbf{A}-\lambda \mathbf{D}) \vec{v}]_{k}=0, \quad k=0, \ldots, n-1,
$$


we get a 5 -term recurrence relation which connects the coordinates $\left\{x_{s}\right\}_{s=k-2}^{k+2}$, $k=0,1,2, \ldots, n-1$ :

$$
\begin{aligned}
& x_{k+2} \frac{(k+2) !}{(k-2) !} \frac{(2 k+\alpha+\beta) !}{(2 k+\alpha+\beta-3) !} \frac{(k+\alpha+\beta+1) !}{(k+\alpha+\beta-1) !}=x_{k+1} \frac{(k+1) !}{(k-2) !} \frac{(2 k+\alpha+\beta-1) !}{(2 k+\alpha+\beta-3) !} \Xi_{1}+ \\
& x_{k}\left(\Xi_{2}-\lambda \frac{(k+1) !}{(k-2) !} \frac{(2 k+\alpha+\beta+4) !}{(2 k+\alpha+\beta-3) !} \frac{(k+\alpha+\beta)}{4}\right)+x_{k-2} \frac{(k+1) !}{(k-1) !} \frac{(2 k+\alpha+\beta+4) !}{(2 k+\alpha+\beta+1) !} \frac{(k+\alpha) !}{(k+\alpha-2) !} \frac{(k+\beta) !}{(k+\beta-2) !} \\
& +\quad x_{k-1} \frac{(2 k+\alpha+\beta+4) !}{(2 k+\alpha+\beta+2) !}\left(k^{2}-1\right)(k+\alpha)(k+\beta)(2 k+\alpha+\beta-1)(\alpha+\beta-2)(\alpha-\beta),
\end{aligned}
$$

where

$$
\begin{aligned}
& \Xi_{1}=(k+\alpha+\beta)(2 k+\alpha+\beta+3)(\alpha+\beta-2)(\alpha-\beta), \Xi_{2}=\frac{k^{4}}{2}+k^{3}(1+\alpha+\beta) \\
& +k^{2} \frac{2 \alpha+2 \beta+2 \alpha^{2}+3 \alpha \beta+2 \beta^{2}+1}{2}+k \frac{(1+\alpha+\beta)\left(\alpha^{2}+\alpha \beta+\beta^{2}\right)}{2}+O_{\alpha, \beta}(1) .
\end{aligned}
$$

This finite difference equation can be considered as a spectral equation for the problem (2.11). We obtain a nontrivial solution of (2.11) if $x_{-1}=x_{-2}=0$ and $x_{n}=x_{n+1}=0$. These boundary conditions will be widely used in the paper. The 5 -term recurrence equation can be rewritten in a matrix form for the bundle $\vec{X}_{k}$ :

$$
\vec{X}_{k+2}=\mathbf{M}_{2}(k, \lambda) \vec{X}_{k}, \quad \vec{X}_{k}:=\left(x_{k-2}, x_{k-1}, x_{k}, x_{k+1}\right)^{T r}, \quad k \in \mathbb{Z}_{+} .
$$

The matrix $\mathbf{M}_{2}$ can be divided in two terms (one is linearly dependent on $\lambda$, the other one is independent on $\lambda): \mathbf{M}_{2}(k, \lambda)=\lambda \mathbf{M}_{2}^{(1)}+\mathbf{M}_{2}^{(0)}$.

The leading coefficients of the expansion of the matrices $\mathbf{M}_{2}^{(1)}$ and $\mathbf{M}_{2}^{(0)}$ are

$$
\mathbf{M}_{2}^{(1)}:=\left(\begin{array}{cccc}
0 & 0 & 0 & 0 \\
0 & 0 & 0 & 0 \\
0 & 0 & (\tilde{b}-8 k-2 a-3) & 0 \\
0 & 0 & 4(2-a)(\alpha-\beta) & (\tilde{b}-16 k-6 a-15)
\end{array}\right)+O\left(\frac{1}{k}\right)
$$

where $a:=\alpha+\beta, \quad \tilde{b}:=-4 k^{2}-4 a k-2 a^{2}$, and

$$
\mathbf{M}_{2}^{(0)}:=\left(\begin{array}{cccc}
0 & 0 & 1 & 0 \\
0 & 0 & 0 & 1 \\
-1-\frac{2}{k} & 0 & 2+\frac{2}{k} & 0 \\
0 & -1-\frac{2}{k} & 0 & 2+\frac{2}{k}
\end{array}\right)+O\left(\frac{1}{k^{2}}\right) .
$$

The relations (2.14) can be rewritten as a finite difference equation involving the vectors $\vec{X}_{k}$ :

$$
\frac{\vec{X}_{k+2}-\vec{X}_{k}}{2 / n}=\frac{n}{2}\left[\mathbf{M}_{2}-\mathbf{I}\right] \vec{X}_{k}, \quad k=0,2, \ldots .
$$

In order to work with a better-structured matrix, we pass from $\vec{X}_{k}$ to $\vec{Y}_{k}$ :

$$
\begin{aligned}
\vec{Y}_{k}:=\mathbf{U}_{k} \vec{X}_{k}, \quad \mathbf{U}_{k} & :=\left(\begin{array}{cccc}
1 & 1 & 0 & 0 \\
1 & -1 & 0 & 0 \\
-k & -k & k & k \\
-k & k & k & -k
\end{array}\right) k>1, \\
\mathbf{U}_{0} & :=\left(\begin{array}{cccc}
1 & 1 & 0 & 0 \\
1 & -1 & 0 & 0 \\
-1 & -1 & 1 & 1 \\
-1 & 1 & 1 & -1
\end{array}\right)
\end{aligned}
$$


satisfying

$$
\vec{Y}_{k+2}=\widehat{\mathbf{M}}_{2}^{(\alpha, \beta)}(k, \lambda) \vec{Y}_{k}, \quad \widehat{\mathbf{M}}_{2}^{(\alpha, \beta)}:=\mathbf{U}_{k+2} \mathbf{M}_{2} \mathbf{U}_{k}^{-1}
$$

Then, we arrive at a finite-difference system, $k=2,4, \ldots$,

$\frac{\vec{Y}_{k+2}-\vec{Y}_{k}}{2 / n}=\frac{n}{k} \mathbf{M}_{3}(k, \lambda) \vec{Y}_{k}, \quad \vec{Y}_{2}=\vec{Y}_{0}+2 \mathbf{M}_{3}(0, \lambda) \vec{Y}_{0}, \quad \vec{Y}_{0}=\left(0,0, C_{1}, C_{2}\right)^{T r}$

where $\mathbf{M}_{3}(0, \lambda)=(1 / 2)\left[U_{2} \mathbf{M}_{2}(0, \lambda) U_{0}^{-1}-\mathbf{I}\right]$ and the matrix $\mathbf{M}_{3}(k, \lambda)$ $=\frac{k}{2}\left[\mathbf{U}_{k+2} \mathbf{M}_{2} \mathbf{U}_{k}^{-1}-\mathbf{I}\right]=\lambda \mathbf{M}_{3}^{(1)}(k)+\mathbf{M}_{3}^{(0)}(k)(k>0)$ has expansions $\mathbf{M}_{3}^{(0)}:=$

$$
\left(\begin{array}{cccc}
0 & 0 & 1 / 2 & 0 \\
0 & 0 & 0 & 1 / 2 \\
2 \alpha(\alpha-2)-\frac{\alpha \diamond(\alpha, \beta)}{k} & \frac{2 \beta(\beta-2)}{k} & 2+\frac{\square(\alpha, \beta)}{2 k} & \frac{\triangle(\alpha, \beta)}{2 k} \\
\frac{2 \alpha(\alpha-2)}{k} & 2 \beta(\beta-2)-\frac{\beta \diamond(\beta, \alpha)}{k} & \frac{\triangle(\beta, \alpha)}{2 k} & 2+\frac{\square(\beta, \alpha)}{2 k}
\end{array}\right)+O\left(\frac{1}{k^{2}}\right),
$$

and

$$
\mathbf{M}_{3}^{(1)}:=\left(\begin{array}{cccc}
0 & 0 & 0 & 0 \\
0 & 0 & 0 & 0 \\
-2 k^{4}-(2 a+10) k^{3} & 2 k^{3} & -2 k^{3} & 2 k^{2} \\
2 k^{3} & -2 k^{4}-(2 a+10) k^{3} & 2 k^{2} & -2 k^{3}
\end{array}\right)+O(k) .
$$

Here we denoted

$$
\begin{aligned}
& \diamond(\alpha, \beta)=4-6 \alpha+2 \alpha^{2}+2 \alpha \beta-\beta, \quad \square(\alpha, \beta)=4 \alpha^{2}-9 \alpha+2 \alpha \beta-\beta+4, \\
& \triangle(\alpha, \beta)=\alpha^{2}-\beta^{2}-2 \alpha+2 \beta+1, \quad \text { and } \quad a:=\alpha+\beta .
\end{aligned}
$$

2.3. General solution of the limiting system of ODEs. Now, we take a formal limit (under an appropriate scaling) of the Finite Difference (FD) problem (2.17) to arrive at a limiting system of ordinary differential equations (ODEs). Indeed, if we denote $\vec{y}=\left(y_{1}, y_{2}, y_{3}, y_{4}\right)^{T r}$ :

$$
\vec{y}(t, l):=\left.\lim _{\substack{n \rightarrow \infty \\ \frac{k}{n} \rightarrow t}} \vec{Y}_{k}(\lambda)\right|_{\lambda=l / n^{4}},
$$

(we shall investigate the existence of this limit later), then we arrive from (2.17) to the system of ODEs:

$$
\frac{d}{d t} \vec{y}(t, l)=\frac{1}{t} \widetilde{\mathbf{M}}_{3}(t, l) \vec{y}(t, l), \quad \widetilde{\mathbf{M}}_{3}(t, l)=\lim _{\frac{k}{n} \rightarrow t} \mathbf{M}_{3}\left(k, \frac{l}{n^{4}}\right),
$$

where

$$
\widetilde{\mathbf{M}}_{3}(t, l)=\left(\begin{array}{cccc}
0 & 0 & 1 / 2 & 0 \\
0 & 0 & 0 & 1 / 2 \\
-2 l t^{4}+2 \alpha(\alpha-2) & 0 & 2 & 0 \\
0 & -2 l t^{4}+2 \beta(\beta-2) & 0 & 2
\end{array}\right) .
$$


Due to the special structure of the main term of asymptotics of the matrix $M_{3}$ in (2.17), this system is split in two second order independent scalar ODEs:

$$
\left\{\begin{array}{l}
t \frac{d}{d t} y_{j}(t)=\frac{z_{j}(t)}{2}, \\
t \frac{d}{d t} z_{j}(t)=2\left[\left(b_{j}\left(b_{j}-2\right)-l t^{4}\right) y_{j}(t)+z_{j}(t)\right],
\end{array} \quad j=1,2,\right.
$$

and

$$
\frac{d^{2}}{d t^{2}} y_{j}(t, l)=\frac{1}{t} \frac{d}{d t} y_{j}(t, l)-\left(t^{2} l-\frac{b_{j}\left(b_{j}-2\right)}{t^{2}}\right) y_{j}, \quad j=1,2 .
$$

Here and in what follows, we use the notation:

$$
b_{j}:=\left\{\begin{array}{l}
\alpha, j=1 \\
\beta, j=2
\end{array} .\right.
$$

The ODE (2.20) is a modified Bessel equation (so-called Bowman form, see 7 , Section 104]); its general solution is

$$
y_{j}(t, l)=\widetilde{C}_{1, j} t J_{\nu\left(b_{j}\right)}\left(\sqrt{l} \frac{t^{2}}{2}\right)+\widetilde{C}_{2, j} t Y_{\nu\left(b_{j}\right)}\left(\sqrt{l} \frac{t^{2}}{2}\right), j=1,2
$$

(we use the notation $\nu($.$) defined in (1.2)). Thus, the general solution of (2.19) is$

$$
\vec{y}(t, l)=\left(y_{1}(t, l), y_{2}(t, l), 2 t y_{1}^{\prime}(t, l), 2 t y_{2}^{\prime}(t, l)\right)^{T r} .
$$

\subsection{Approximate general solution of the FD problem and further plan.}

Thus, in the regime

$$
\left\{\begin{array}{l}
n \rightarrow \infty \\
\frac{k}{n} \rightarrow t \in K \Subset(0,1] \quad,
\end{array}\right.
$$

the general solutions (2.22) could be a good approximation for general solutions $C_{1} \vec{Y}_{k}^{(1)}+C_{2} \vec{Y}_{k}^{(2)}$ of (2.15) - (2.14), for $k \in \mathbb{Z}$ :

$$
\vec{Y}_{k}^{(1)}(\lambda) \approx\left(\begin{array}{c}
y_{1}\left(\frac{k}{n}, \lambda n^{4}\right) \\
0 \\
\frac{2 k}{n} y_{1}^{\prime}\left(\frac{k}{n}, \lambda n^{4}\right) \\
0
\end{array}\right), \quad \vec{Y}_{k}^{(2)}(\lambda) \approx\left(\begin{array}{c}
0 \\
y_{2}\left(\frac{k}{n}, \lambda n^{4}\right) \\
0 \\
\frac{2 k}{n} y_{2}^{\prime}\left(\frac{k}{n}, \lambda n^{4}\right)
\end{array}\right)
$$

here $\left({ }^{\prime}\right)$ denotes the derivative with respect to the first variable.

Now, we recall (see Introduction) the further steps we need to proceed in order to obtain in the regime (2.23) asymptotics of the exact constant (2.9) and the vector $\vec{v}$ - 2.11) which defines the extremal polynomial $Q_{n}^{\prime}$. To choose, from the general (approximate) solution (2.24) for $k \in \mathbb{Z}$, a solution which corresponds to the boundary conditions $(\mathrm{BC})$

$$
v_{-2}=v_{-1}=0 \quad \text { and } \quad v_{n}=v_{n+1}=0,
$$

we proceed as in [14]. First we find a set of two particular solutions of (2.14) $x_{k}(\lambda)$ for $\lambda=0$ which correspond to the boundary conditions (2.25) at the left end:

$$
x_{-2}^{(j)}, x_{-1}^{(j)}=0, \quad j=1,2 .
$$

The second step is to choose constants $C_{1}, C_{2}$ for (2.24) such that asymptotics of 
$y_{j}(t, l), j=1,2$, when $t \rightarrow 0$, would match with asymptotics of $\vec{Y}_{k}^{(j)}(0) j=1,2$ when $k \rightarrow \infty$, which correspond to the particular solutions $x_{k}^{(j)}(0)$. It defines the unknown constants for (2.24), (2.22). The last step is to obtain a linear combination of two approximate discrete solutions $\widetilde{v}_{k}^{(j)}(\lambda), j=1,2$ (we get them from (2.24)), satisfying the left end $\mathrm{BC}$ in (2.25), such that this combination satisfies the right end $\mathrm{BC}$ in (2.25). It is possible to do this if

$$
\operatorname{det}\left|\begin{array}{cc}
\widetilde{v}_{n}^{(1)}(\lambda) & \widetilde{v}_{n+1}^{(1)}(\lambda) \\
\widetilde{v}_{n}^{(2)}(\lambda) & \widetilde{v}_{n+1}^{(2)}(\lambda)
\end{array}\right|=0 .
$$

This equation (in $\lambda$ ) has the same meaning as (2.10) and its solutions with approximate $\widetilde{v}_{n}^{(j)}(\lambda)$ gives an approximation of the eigenvalues $\lambda(\mathbf{A}, \mathbf{D})$.

2.5. Two particular solutions of the FD problem for $\lambda=0$. Here we find solutions of the recurrence equation associated with (2.13) for $\lambda=0$, satisfying the $\mathrm{BC}(2.25)$ at the left end

$$
v_{-2}=v_{-1}=0 \text {. }
$$

We are looking for the solutions of recurrences

$$
\left[\mathbf{C}_{1}^{T r} \mathbf{C}_{2}^{T r} \mathbf{N}^{-1} \mathbf{D}^{+} \mathbf{N}^{-1} \mathbf{C}_{2} \mathbf{C}_{1} \vec{v}\right]_{k}=0, \quad k=0,1, \ldots, n-3 .
$$

First we consider the equation

$$
\left[\mathbf{C}_{2} \vec{h}\right]_{k}=0, \text { with }\left(\mathbf{C}_{2} \vec{h}\right)_{k-1}=h_{k-1}+\frac{2 k(k+\alpha+1) h_{k}}{(2 k+\alpha+\beta+1)(2 k+\alpha+\beta+2)}
$$

The structure of this equation is such that $h_{-1}=0$ and $h_{-2}$ can take any values, and we can put it to be zero. It is easy to check that the homogeneous equation (2.30) has a solution

$$
h_{-2}=h_{-1}=0, \quad h_{k}=\frac{(2 k+\alpha+\beta+2) !}{(-2)^{k} k !(k+\alpha+1) !}, \quad k=0,1, \ldots, n-1 .
$$

Next, we consider the equation:

$$
\left[\mathbf{C}_{1} \vec{h}\right]_{k}=0, \text { with }\left(\mathbf{C}_{1} \vec{h}\right)_{k-1}=h_{k-1}+\frac{-2 k(k+\beta)}{(2 k+\alpha+\beta)(2 k+\alpha+\beta+1)} h_{k} .
$$

Again we can check that this homogeneous equation has a solution

$$
h_{-2}=h_{-1}=0, \quad h_{k}=\frac{(2 k+\alpha+\beta+1) !}{2^{k} k !(k+\beta) !}, \quad k=0,1, \ldots, n-1,
$$

which can be taken as the first particular solution of (2.29), (2.28):

$$
v_{k}^{(1)}(0)=\frac{(2 k+\alpha+\beta+1) !}{2^{k} k !(k+\beta) !}, \quad k=0,1, \ldots, n-1 .
$$

We find the second particular solution of (2.29), (2.28) as a solution of the nonhomogeneous equation (2.32) with right hand side $h_{k-1}$ from (2.31)

$$
v_{k-1}^{(2)}(0)-\frac{2 k(k+\beta)}{(2 k+\alpha+\beta)(2 k+\alpha+\beta+1)} v_{k}^{(2)}(0)=\frac{-2 k(2 k+\alpha+\beta) !}{(-2)^{k} k !(k+\alpha) !} .
$$

It is easy to check that this nonhomogeneous equation has a solution

$$
v_{k}^{(2)}(0)=\frac{(2 k+\alpha+\beta+1) !}{(-2)^{k} k !(k+\alpha) !}, \quad k=0,1, \ldots, n-1 .
$$


Note that for $v^{(1)}, v^{(2)}$ one has

$$
\mathbf{C}_{1}^{T r} \mathbf{C}_{2}^{T r} \mathbf{N}^{-1} \mathbf{D}^{+} \mathbf{N}^{-1} \mathbf{C}_{2} \mathbf{C}_{1} \vec{v}=\text { const }_{1} \vec{e}_{n-1}+\text { const }_{2} \vec{e}_{n-2} .
$$

where $\vec{e}_{n-2}$ and $\vec{e}_{n-1}$ are the following vectors of $\mathbb{R}^{n}: \vec{e}_{n-2}=(0, \ldots, 0,1,0)^{T r}$, $\vec{e}_{n-1}=(0, \ldots, 0,0,1)^{T r}$.

2.6. Matching of the particular solutions of FD and ODEs problems. We obtain the particular solutions $\vec{X}_{k}^{(1)}(0), \vec{X}_{k}^{(2)}(0)$ of (2.14) from (2.33), (2.34), (2.12):

$$
x_{k}^{(1)}(0):=\frac{(k+\alpha) !}{k !}, \quad x_{k}^{(2)}(0):=\frac{(k+\beta) !}{(-1)^{k} k !} .
$$

Using (2.15) we have

$$
\vec{Y}_{k}^{(j)}(0)=\left(\begin{array}{l}
x_{k-2}^{(j)}+x_{k-1}^{(j)} \\
x_{k-2}^{(j)}-x_{k-1}^{(j)} \\
k\left(-\left(x_{k-2}^{(j)}+x_{k-1}^{(j)}\right)+\left(x_{k}^{(j)}+x_{k+1}^{(j)}\right)\right) \\
k\left(-\left(x_{k-2}^{(j)}-x_{k-1}^{(j)}\right)+\left(x_{k}^{(j)}-x_{k+1}^{(j)}\right)\right)
\end{array}\right), \quad j=1,2 .
$$

Substituting the expansions of (2.35) in (2.36), we get for $\vec{Y}_{k}^{(1)}(0)$ and $\vec{Y}_{k}^{(2)}(0)$

$$
k^{\alpha}\left[\left(\begin{array}{c}
2+\frac{(\alpha-2) \alpha}{k} \\
-\frac{\alpha}{k} \\
4 \alpha+O\left(\frac{1}{k}\right) \\
O\left(\frac{1}{k}\right)
\end{array}\right)+O\left(\frac{1}{k^{2}}\right)\right], \quad k^{\beta}\left[\left(\begin{array}{c}
-\frac{\beta}{k} \\
2+\frac{(\beta-2) \beta}{k} \\
O\left(\frac{1}{k}\right) \\
4 \beta+O\left(\frac{1}{k}\right)
\end{array}\right)+O\left(\frac{1}{k^{2}}\right)\right],
$$

correspondingly. We conclude for $k \rightarrow \infty, j=1,2$ (recalling the notation (2.21)):

$$
\vec{Y}_{k}^{(j)}(0)=k^{b_{j}}\left(\vec{C}_{0}^{(j)}+O\left(\frac{1}{k}\right)\right), \vec{C}_{0}^{(1)}:=\left(\begin{array}{c}
2 \\
0 \\
4 \alpha \\
0
\end{array}\right), \vec{C}_{0}^{(2)}:=\left(\begin{array}{c}
0 \\
2 \\
0 \\
4 \beta
\end{array}\right) .
$$

Now, we state a "Matching Condition" for the choice of the particular solutions of the differential problem (2.19)-(2.22) when $t \rightarrow 0$ :

$$
\vec{y}^{(j)}(t, l)=t^{b_{j}}\left(\vec{C}_{0}^{(j)}+o(1)\right) .
$$

If this condition is satisfied, then, for $\lambda=l n^{-4}$, we expect that in the regime (2.23)

$$
\vec{Y}_{k}^{(j)}\left(l n^{-4}\right)=n^{b_{j}} \vec{y}^{(j)}\left(\frac{k}{n}, l\right)+o\left(k^{b_{j}}\right) .
$$

This assertion will be proved later (see Theorem 3.1).

Using the well-known power series expansion of the Bessel functions for $x \rightarrow 0$ (see [7]),

$$
J_{\nu}(x)=\hat{c}_{\nu} x^{\nu}\left(1+\tilde{c}_{\nu} x^{2}+O\left(x^{4}\right)\right), \quad \hat{c}_{\nu}:=\frac{1}{2^{\nu} \nu !},
$$

and matching the conditions (2.38), (2.37), we obtain expressions for the constants $\tilde{C}_{k, j}$ in the presentation (2.22) of the general solution of (2.19):

$$
\tilde{C}_{2, j}=0, \quad \tilde{C}_{1, j}=2^{\nu_{j}+1}\left(\hat{c}_{\nu_{j}} l^{\nu_{j} / 2}\right)^{-1}, \quad \nu_{j}=\nu\left(b_{j}\right), \quad j=1,2 .
$$


Thus, the particular solutions of (2.19), satisfying the condition (2.38), are

$$
\vec{y}^{(1)}(t, l)=\left(\begin{array}{c}
y_{1}(t, l) \\
0 \\
2 t \frac{d}{d t} y_{1}(t, l) \\
0
\end{array}\right), \quad \vec{y}^{(2)}(t, l)=\left(\begin{array}{c}
0 \\
y_{2}(t, l) \\
0 \\
2 t \frac{d}{d t} y_{2}(t, l)
\end{array}\right),
$$

where

$$
y_{j}(t, l)=\frac{2^{b_{j}} \nu_{j} !}{l^{\nu_{j} / 2}} t J_{\nu_{j}}\left(\frac{\sqrt{l} t^{2}}{2}\right), \quad j=1,2 .
$$

2.7. Matching of the right end BC for $\left\{v_{k}^{(j)}\right\}_{k=0}^{n-1}$. Now, substituting (2.40)(2.41) into (2.39), (2.15), and (2.12), we arrive at the two particular approximate sequences $\left\{v_{k}^{(s)}(\lambda)\right\}_{k \in \mathbb{Z}_{+}}$, satisfying the left end BC in (2.25):

$$
\left.v_{n}^{(j)}(\lambda)\right|_{\lambda=\frac{l}{n^{4}}}=(-1)^{(j-1) n} \frac{(2 n+\alpha+\beta) ! \nu_{j} ! n^{b_{j}}}{2^{n-2 \nu_{j}}(n+\alpha) !(n+\beta) ! l^{\nu_{j} / 2}}\left(J_{\nu_{j}}\left(\frac{\sqrt{l}}{2}\right)+o(1)\right) \text {. }
$$

Thus, the right end BC (see (2.27) ) when $n \rightarrow \infty$ and $\lambda=\frac{l}{n^{4}}$, is equivalent to

$$
\left.C(l, n)\left(\operatorname{det}\left|\begin{array}{cc}
J_{\nu(\alpha)}\left(\frac{\sqrt{l}}{2}\right) & J_{\nu(\alpha)}\left(\frac{\sqrt{l}}{2}\right) \\
-J_{\nu(\beta)}\left(\frac{\sqrt{l}}{2}\right) & J_{\nu(\beta)}\left(\frac{\sqrt{l}}{2}\right)
\end{array}\right|+o(1)\right)\right)=0, \quad C(l, n) \neq 0, l>0 .
$$

From here we conclude that the roots of the equation

$$
J_{\nu(\alpha)}\left(\frac{\sqrt{l}}{2}\right) J_{\nu(\beta)}\left(\frac{\sqrt{l}}{2}\right)+o(1)=0
$$

give approximate values of $\lambda=\frac{l}{n^{4}}$, for which the BC (2.25) are fulfilled. Indeed, if $\alpha<\beta$ and $l_{0}$ is the smallest zero of the Bessel function $J_{\nu(\alpha)}\left(\frac{\sqrt{l}}{2}\right)$, then the determinant in (2.27) changes the sign from $l_{0}-\epsilon$ to $l_{0}+\epsilon$. This gives a correct interval for the smallest $\lambda$ for which (2.27) vanishes. In the case $\alpha=\beta$ the equation (2.42) takes the form $J_{\nu(\alpha)}^{2}\left(\frac{\sqrt{l}}{2}\right)+o(1)=0$. If $l_{0}$ is the smallest zero of the Bessel function $J_{\nu(\alpha)}\left(\frac{\sqrt{l}}{2}\right)$, then by Rouché's theorem the determinant (2.27) has two zeros in the complex plane in the neighborhood of $l_{0}$. By symmetry of matrices of generalized eigenvalue problem (2.10) these zeros have to be real. This gives a correct interval for the smallest $\lambda$ for which (2.27) vanishes in the case $\alpha=\beta$. Therefore the minimal root $j_{\nu}$ of the Bessel function $J_{\nu}$ gives the main term of asymptotics of the exact Markov-Bernstein constant

$$
M_{n}=\frac{n^{2}}{2 j_{\nu^{*}}}(1+o(1)) .
$$

\section{Matching And CONVERGEnce of FD And DE PROBlems}

3.1. Statements of the results. Our derivation of the asymptotics (2.43) contained one assumption which requires a special rigorous treatment. It is the convergence in the regime (2.23) of the discrete solution to the continuous solution, see (2.39). Here we state a theorem which establishes (2.39) under a restriction on $(\alpha, \beta)$. 
Theorem 3.1. Let $\left\{\vec{Y}_{k}^{(j)}(\lambda)\right\}_{k=0}^{\infty}, j=1,2$, be a set of the particular solutions of the FD problem, i.e. the recurrence (2.16) with the matrix $\widehat{\mathbf{M}}_{2}^{(\alpha, \beta)}(k, \lambda)$ such that $\left\{\vec{Y}_{k}^{(j)}(\lambda)\right\}_{k=0}^{\infty}, j=1,2$, are given by (2.36), 2.35), $\vec{Y}_{0}^{(j)}(\lambda)=\vec{Y}_{0}^{(j)}(0)$.

Let the parameters $(\alpha, \beta)$ in $\widehat{\mathbf{M}}_{2}^{(\alpha, \beta)}$ satisfy the condition:

$$
|\alpha-\beta|<4, \quad \alpha, \beta>-1 .
$$

Then, for $\lambda=\frac{l}{n^{4}}, \frac{k}{n} \rightarrow t$ and $n \rightarrow \infty$, uniformly for $l \in \widetilde{K} \Subset \mathbb{C}, t \in K \Subset(0,1]$,

$$
\vec{Y}_{k}^{(j)}(\lambda)=n^{b_{j}}\left(\vec{y}^{(j)}(t, l)+o\left(t^{b_{j}}\right)\right), \quad b_{j}=\left\{\begin{array}{cc}
\alpha, & j=1 \\
\beta, & j=2
\end{array},\right.
$$

holds true. Here $\vec{y}^{(j)}, j=1,2$, are the particular solutions (2.41), (2.40) of the DE problem (2.19) satisfying the matching condition (2.38).

Taking into account the procedure of derivation of (2.43) we obtain the validity of Theorem 1.1 as a corollary of Theorem 3.1 .

3.2. Proof of Theorem 3.1. In our proof we use an approach proposed in 14. The comparison of $\vec{Y}_{k}^{j}$ and $n^{b_{j}} \vec{y}^{(j)}$, in the regime (2.23), will be performed by means of the following relations. In what follows we denote by $|v|$ a norm of the vector $v$ in the vector space $\mathbb{R}^{n}$ and by $\|S\|$ the associated matrix norm. For two recurrent sequences $\left\{\vec{v}_{k}\right\},\left\{\vec{w}_{k}\right\}$, defined by

$$
\vec{v}_{k+2}=S_{k}^{(1)} \vec{v}_{k}, \quad \vec{w}_{k+2}=S_{k}^{(2)} \vec{w}_{k}, \quad \vec{v}_{k}, \vec{w}_{k} \in \mathbb{R}^{N}, \quad k \in \mathbb{N} * 2,
$$

we have for $k>k_{0}$

$$
\begin{gathered}
\left|\vec{w}_{k}-\vec{v}_{k}\right| \leqslant \\
{\left[\left|\vec{w}_{k_{0}}-\vec{v}_{k_{0}}\right|+\sum_{m=k_{0}, 2}^{k-2}\left|\left(S_{m}^{(2)}-S_{m}^{(1)}\right) \vec{v}_{m}\right| E\left(k_{0}, m, S^{(2)}\right)\right] E^{-1}\left(k_{0}, k-2, S^{(2)}\right),}
\end{gathered}
$$

where the summation is performed with Step 2, and

$$
E\left(k_{0}, m, S\right):=\exp \left(\sum_{i=k_{0}, 2}^{m}\left(1-\left\|S_{i}\right\|\right)\right) .
$$

The estimate (3.3) easily follows by induction from

$$
\left|\vec{w}_{n+2}-\vec{v}_{n+2}\right|=\left|S_{n}^{(2)} \vec{w}_{n}-S_{n}^{(1)} \vec{v}_{n}\right| \leqslant\left\|S_{n}^{(2)}\right\|\left|\vec{w}_{n}-\vec{v}_{n}\right|+\left|\left(S_{n}^{(2)}-S_{n}^{(1)}\right) \vec{v}_{n}\right|
$$

and from the inequality $x e^{1-x} \leqslant 1$ applied to $\left\|S_{n}^{(2)}\right\|$.

1) We are going to use the estimate (3.3) to compare the solution $\vec{Y}_{k}^{(j)}(\lambda)$ of the finite difference problem (2.17) with the solution $\vec{y}^{(j)}(t, l)$ (see (2.40)-(2.41)) of the differential problem (2.19). To do this (having in mind (3.3) ) we define a difference operator which connects values of $\vec{y}^{(j)}(t)$ taken on the discrete grid $t:=\frac{k}{n}, k \in \mathbb{N}_{+}$:

$$
\vec{y}^{(j)}\left(\frac{k+2}{n}, l\right)=\widetilde{\widetilde{\mathbf{M}}}_{2}(k, l, n) \vec{y}^{(j)}\left(\frac{k}{n}, l\right), \quad \widetilde{\widetilde{\mathbf{M}}}_{2}=:\left(\mathbf{I}+\frac{2}{k} \widetilde{\widetilde{M}}_{3}\right) .
$$

We apply the following lemma from [14] (see Lemma 3.2). 
Lemma 3.2. Let $M(t)$ be a matrix-valued function solving the Cauchy problem with smooth matrix-valued coefficient $F$ :

$$
\frac{d}{d t} M(t)=F(t) M(t), \quad M\left(t_{1}\right)=\mathbf{I} .
$$

Then the following estimate holds for $t_{2}>t_{1}, \int_{t_{1}}^{t_{2}}\|F(t)\| d t<1$ :

$$
\left\|M\left(t_{2}\right)-\mathbf{I}-\left(t_{2}-t_{1}\right) F\left(t_{1}\right)\right\|<2\left(t_{2}-t_{1}\right)^{2} \max _{\left[t_{1} ; t_{2}\right]}\left\|F^{\prime}(t)+F^{2}(t)\right\| .
$$

Applying this lemma to $M=\widetilde{\widetilde{\mathbf{M}}}_{2}, t_{1}=\frac{k}{n}, t_{2}=\frac{k+2}{n}$ we get an estimate which we shall use in our analysis later.

$$
\left\|\widetilde{\widetilde{\mathbf{M}}}_{3}(k, l, n)-\widetilde{\mathbf{M}}_{3}\left(\frac{k}{n}, l\right)\right\|=O\left(\frac{1}{k}\right),
$$

here $\widetilde{\mathbf{M}}_{3}$ is defined in (2.19) and $k \rightarrow \infty, \frac{k}{n} \in K_{1} \Subset \mathbb{R}_{+}, l \in K_{2} \Subset \mathbb{C}$.

2) Now, we can rewrite (3.3) for our purpose

$$
\begin{aligned}
& \left|\vec{Y}_{k}^{(j)}(\lambda)-n^{b} \vec{y}^{(j)}\left(\frac{k}{n}, \lambda n^{4}\right)\right| \lesssim\left\{\left|\vec{Y}_{k_{0}}^{(j)}(\lambda)-n^{b} \vec{y}^{(j)}\left(\frac{k_{0}}{n}, \lambda n^{4}\right)\right|\right. \\
& * \sum_{m=k_{0}, 2}^{k-2}\left|\left(\widehat{\mathbf{M}}_{2}^{(\alpha, \beta)}(m, \lambda)-\widetilde{\widetilde{\mathbf{M}}}_{2}\left(m, \lambda n^{4}\right)\right) n^{b} \vec{y}^{(j)}\left(\frac{k}{n}, \lambda n^{4}\right)\right| \\
& \left.* E\left(k_{0}, m, \widehat{\mathbf{M}}_{2}^{(\alpha, \beta)}\right)\right\} E^{-1}\left(k_{0}, k-2, \widehat{\mathbf{M}}_{2}^{(\alpha, \beta)}\right),
\end{aligned}
$$

where $\widehat{\mathbf{M}}_{2}, \widetilde{\widetilde{\mathbf{M}}}_{2}$ are defined in (2.16), (3.4), correspondingly.

3) To proceed with (3.6) we start with an estimation of the initial deviation. We put $k_{0}:=n^{1-\delta}$, where $\delta>0$ will be fixed later. We shall compare the initial data in (3.6), estimating their deviation from the corresponding particular solution $Y_{k_{0}}^{(j)}(0)$, which we know explicitly (2.36), (2.35).

$$
\begin{aligned}
& \left|\vec{Y}_{k_{0}}^{(j)}(\lambda)-n^{b} \vec{y}^{(j)}\left(\frac{k_{0}}{n}, \lambda n^{4}\right)\right|< \\
& \left|\vec{Y}_{k_{0}}^{(j)}(\lambda)-\vec{Y}_{k_{0}}^{(j)}(0)\right|+\left|\vec{Y}_{k_{0}}^{(j)}(0)-n^{b} \vec{y}^{(j)}\left(\frac{k_{0}}{n}, \lambda n^{4}\right)\right|, \quad k_{0}=n^{1-\delta} .
\end{aligned}
$$

To estimate the second term in the right hand side of (3.7) we use the matching condition (2.38)

$$
\begin{aligned}
& \left|\vec{Y}_{k_{0}}^{(j)}(0)-n^{b_{j}} \vec{y}^{(j)}\left(\frac{k_{0}}{n}, l\right)\right| \\
& =\left|\left(\vec{C}_{0}^{(j)} k_{0}^{b_{j}}+O\left(\frac{1}{k_{0}}\right)\right)-\left(\vec{C}_{0}^{(j)} k_{0}^{b_{j}}+O\left(\frac{k_{0}^{4}}{n^{4}}\right)\right)\right| \\
& =O\left(k_{0}^{b-1}\right)+O\left(k_{0}^{b}\left(\frac{k_{0}}{n}\right)^{4}\right) .
\end{aligned}
$$


To estimate the first term in the right hand side of (3.7) we again use (3.3)

$$
\begin{aligned}
\left|\vec{Y}_{k_{0}}^{(j)}(\lambda)-\vec{Y}_{k_{0}}^{(j)}(0)\right| & \leqslant\left\{\left|\vec{Y}_{2}^{(j)}(\lambda)-\vec{Y}_{2}^{(j)}(0)\right|\right. \\
* \sum_{m=2,2}^{k_{0}-2} \mid & \left(\widehat{\mathbf{M}}_{2}^{(\alpha, \beta)}(m, \lambda)-\widehat{\mathbf{M}}_{2}^{(\alpha, \beta)}(m, 0)\right) \vec{Y}_{m}^{(j)}(0) \mid \\
& \left.* E\left(k_{0}, m, \widehat{\mathbf{M}}_{2}^{(\alpha, \beta)}(m, \lambda)\right)\right\} E^{-1}\left(k_{0}, m, \widehat{\mathbf{M}}_{2}^{(\alpha, \beta)}(m, \lambda)\right) .
\end{aligned}
$$

For further estimations we shall use \|\|$_{\delta}$-norm introduced in [14. This norm is related to a basis in which the operator $\mathbf{M}_{3}^{(0)}(\infty)$ defined in (2.16), (2.17) has a matrix (with eigenvalues on the diagonal) which is arbitrarily close to a diagonal matrix

$$
\lim _{m \rightarrow \infty} \widehat{\mathbf{M}}_{2}^{(\alpha, \beta)}(m, 0)=: \widehat{\mathbf{M}}_{2}^{(\alpha, \beta)}(\infty, 0)=\left(\mathbf{I}+\frac{1}{k} \mathbf{M}_{3}^{(0)}(\infty)\right)
$$

In such a basis the operator $\widehat{\mathbf{M}}_{2}^{(\alpha, \beta)}(\infty, 0)$ will have a norm close to $\left(1+\frac{1}{k} B\right)$, where

$$
B:=\max \{\alpha, 2-\alpha, \beta, 2-\beta\}
$$

is the maximal real part of eigenvalues of the matrix

$$
\mathbf{M}_{3}^{(0)}(\infty):=\widetilde{\mathbf{M}}_{3}(0,0)=\left(\begin{array}{cccc}
0 & 0 & 1 / 2 & 0 \\
0 & 0 & 0 & 1 / 2 \\
2 \alpha(\alpha-2) & 0 & 2 & 0 \\
0 & 2 \beta(\beta-2) & 0 & 2
\end{array}\right)
$$

That is, for each $\delta>0$ there exist $k_{\delta} \in \mathbb{N}$ and a basis such that, with respect to the Euclidean norm $|\quad|_{\delta}$ associated with this basis (see 14 for the details about the $\delta$-norm), the corresponding operator norm \|\|$_{\delta}$ has the estimate

$$
\forall k>k_{\delta}, \quad\left\|\widehat{\mathbf{M}}_{2}^{(\alpha, \beta)}(k, 0)\right\|=\left\|\mathbf{I}+\frac{1}{k} \mathbf{M}_{3}^{(0)}(k)\right\|_{\delta}<1+\frac{B+\delta}{k} .
$$

Then, we estimate the terms from the right hand side of (3.9). We have (see (2.17))

$$
\begin{gathered}
\left|\left(\widehat{\mathbf{M}}_{2}^{(\alpha, \beta)}(m, \lambda)-\widehat{\mathbf{M}}_{2}^{(\alpha, \beta)}(m, 0)\right) \vec{Y}_{m}^{(j)}(0)\right|_{\delta} \\
=\frac{1}{m}\left|\left(\mathbf{M}_{3}(m, \lambda)-\mathbf{M}_{3}(m, 0)\right) \vec{Y}_{m}^{(j)}(0)\right|_{\delta}=\frac{1}{m}\left|\mathbf{M}_{3}^{(1)} \lambda \vec{Y}_{m}^{(j)}(0)\right| \leqslant O\left(|\lambda| \frac{m^{4}}{m} m^{b_{j}}\right) .
\end{gathered}
$$

Analogously

$$
\left\|\mathbf{M}_{3}(m, \lambda)-\mathbf{M}_{3}(m, 0)\right\|_{\delta}=|\lambda|\left\|\mathbf{M}_{3}^{(1)}\right\|=O\left(\lambda m^{4}\right),
$$

and

$$
\begin{gathered}
\left\|\mathbf{I}+\frac{1}{m} \mathbf{M}_{3}(m, \lambda)\right\|_{\delta} \leqslant \\
=1+\frac{B+\delta}{m}+\frac{1}{m}\left\|\mathbf{M}_{3}(m, \lambda)-\mathbf{M}_{3}(m, 0)\right\|_{\delta} \\
m
\end{gathered}
$$


Now, we proceed with (3.9). For $k=k_{0}=n^{1-\delta}$ we have

$$
\begin{aligned}
&\left|\vec{Y}_{k}^{(j)}(\lambda)-\vec{Y}_{k}^{(j)}(0)\right| \leqslant O(1) \exp \left\{\sum_{\tilde{k}=2,2}^{k-2} \frac{B+\delta+O\left(|l| n^{-4}\right) \tilde{k}^{4}}{\tilde{k}}\right\} \\
& * \sum_{\tilde{k}=2,2}^{k-2} \frac{O\left(|l| n^{-4}\right) \tilde{k}^{4+b_{j}}}{k} \exp \left\{-\sum_{\tilde{\tilde{k}}=2,2}^{\tilde{k}-2} \frac{B+\delta+O\left(|l| n^{-4}\right) \tilde{\tilde{k}}^{4}}{\tilde{\kappa}}\right\} \\
&=\frac{|l|}{n^{4}} O\left(k^{B+\delta} \exp \left[O\left(|l|\left(\frac{k}{n}\right)^{4}\right)\right]\right) \sum_{\tilde{k}=2,2}^{k-2} \tilde{k}^{4+b_{j}-B-\delta-1} \exp \left[O\left(|l|\left(\frac{\tilde{k}}{n}\right)^{4}\right)\right] .
\end{aligned}
$$

At this point we assume that

$$
4+b_{j}>B+\delta
$$

This assumption (see (3.10) ) implies the restriction (3.1) in our theorems. Thus, we obtained

$$
\left|\vec{Y}_{k_{0}}^{(j)}(\lambda)-\vec{Y}_{k_{0}}^{(j)}(0)\right| \leqslant O\left(\left(\frac{k_{0}}{n}\right)^{4} k_{0}^{b_{j}}\right),
$$

and finally, for the initial deviation (3.7) in (3.6) we get from here and (3.8)

$$
\left|\vec{Y}_{k_{0}}^{(j)}(\lambda)-n^{b_{j}} \vec{y}\left(\frac{k_{0}}{n}, \lambda n^{4}\right)\right| \leqslant O\left(k_{0}^{b_{j}-1}\right)+O\left(\left(\frac{k_{0}}{n}\right)^{4} k_{0}^{b_{j}}\right) .
$$

4) Now, we come back to (3.6). Using (3.5) and the triangle inequality one has

$$
\begin{gathered}
k\left\|\widetilde{\widetilde{\mathbf{M}}}_{2}\left(k, \lambda n^{4}\right)-\widehat{\mathbf{M}}_{2}^{(\alpha, \beta)}(k, \lambda)\right\|_{\delta}=\left\|\widetilde{\widetilde{\mathbf{M}}}_{3}(k, l, n)-\mathbf{M}_{3}\left(k, \frac{l}{n^{4}}\right)\right\|_{\delta} \\
\leqslant O\left(\frac{1}{k}\right)+\frac{l}{n^{4}}\left(k^{4}+\text { const } k^{3}+\ldots\right)-\frac{l}{n^{4}} k^{4}=O\left(\frac{1}{k}\right)+O\left(\frac{l}{n^{4}} k^{3}\right) \\
=o\left(\left(\frac{k}{n}\right)^{4} k^{-\varepsilon_{1}}\right)+o\left(k^{-\varepsilon}\right), \quad \forall \varepsilon, \varepsilon_{1} \in(0,1) .
\end{gathered}
$$

Substituting this estimate and (3.12), (3.11) in (3.6), we proceed:

$$
\begin{gathered}
\left|n^{b_{j}} \vec{y}^{(j)}\left(\frac{k}{n}, \lambda n^{4}\right)-\vec{Y}_{k}^{(j)}(\lambda)\right| \leqslant\left\{O\left(k_{0}^{b_{j}-1}\right)+O\left(k_{0}^{b_{j}}\left(\frac{k_{0}}{n}\right)^{4}\right)\right. \\
\left.+\sum_{m=k_{0}, 2}^{k-2} \frac{1}{m}\left[o\left(m^{-\varepsilon_{1}}\left(\frac{m}{n}\right)^{4}\right)+o\left(m^{-\varepsilon}\right)\right] n^{b_{j}} O\left(\frac{m}{n}\right)^{b_{j}} \exp \left(-\sum_{s=k_{0}, 2}^{m} \frac{B+\delta+O\left(|l| n^{-4} s^{4}\right)}{s}\right)\right\} \\
* \exp \left(\sum_{s=k_{0}, 2}^{m} \frac{B+\delta+O\left(|l| n^{-4} s^{4}\right)}{s}\right) .
\end{gathered}
$$


Assuming again from (3.1)

$$
4+b_{j}>B+\delta+\varepsilon_{1}
$$

we continue:

$$
\begin{gathered}
\left|n^{b} \vec{y}^{(j)}\left(\frac{k}{n}, \lambda n^{4}\right)-\vec{Y}_{k}^{(j)}(\lambda)\right| \leqslant\left\{o\left(k_{0}^{b_{j}-\tilde{\varepsilon}}\right)+o\left(k_{0}^{b_{j}}\left(\frac{k_{0}}{n}\right)^{B+\delta-b_{j}}\right)\right. \\
\left.+\sum_{m=k_{0}, 2}^{k} \frac{1}{m}\left[o\left(m^{b-\varepsilon_{1}}\left(\frac{m}{n}\right)^{4}\right)+o\left(m^{b-\varepsilon}\right)\right] *\left(\frac{k_{0}}{m}\right)^{B+\delta} \exp \left(O\left[\left(\frac{m}{n}\right)^{4}-\left(\frac{k_{0}}{n}\right)^{4}\right]\right)\right\} \\
*\left(\frac{k}{k_{0}}\right)^{B+\delta} \exp \left(O\left[\left(\frac{k}{n}\right)^{4}-\left(\frac{k_{0}}{n}\right)^{4}\right]\right) \leqslant\left[o\left(k_{0}^{b_{j}-\tilde{\varepsilon}}\right)\left(\frac{k}{k_{0}}\right)^{B+\delta}+o\left(n^{b_{j}}\right)\right. \\
\left.+\sum_{m=k_{0}, 2}^{k-2} \frac{1}{m}\left[o\left(m^{b_{j}-\varepsilon_{1}}\left(\frac{m}{n}\right)^{4}\right)+o\left(m^{b_{j}-\varepsilon}\right)\right]\left(\frac{k}{m}\right)^{B+\delta} \exp (O(1))\right] \exp (O(1)) \\
\leqslant o\left(n^{b}\right)+o\left(k_{0}^{b_{j}-\tilde{\varepsilon}}\left(\frac{k}{k_{0}}\right)^{B+\delta}\right)+o\left(k^{b_{j}-\varepsilon_{1}}\right)+o\left(k_{0}^{b_{j}-\tilde{\varepsilon}}\left(\frac{k}{k_{0}}\right)^{B+\delta}\right) .
\end{gathered}
$$

Taking into account that

$$
k_{0}=n^{1-\delta}, \quad \frac{k}{n}=t \in K \Subset \mathbb{R}_{+},
$$

we see that the right hand side of this estimation is equal to

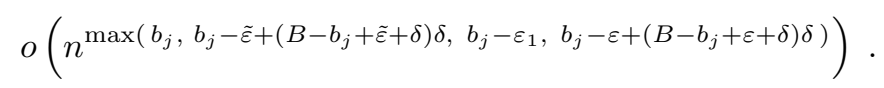

Since $\tilde{\varepsilon}<\varepsilon$, we choose $\delta$ :

$$
\delta(B-b+\tilde{\varepsilon}+\delta)<\tilde{\varepsilon},
$$

which yields

$$
\left|n^{b_{j}} \vec{y}^{(j)}\left(\frac{k}{n}, l\right)-\vec{Y}_{k}^{(j)}\left(\frac{l}{n^{4}}\right)\right|=o\left(n^{b_{j}}\right) .
$$

Theorem is proved.

The authors are very grateful to the referee for careful reading of the manuscript and very important and valuable remarks.

\section{REFERENCES}

[1] A.A. Markov, On one question of D.I. Mendeleev, Izvestiya Peterburg Akademii Nauk, 62, (1889), 1-24 (in Russian).

[2] S.N. Bernshtein, On the best approximation of the continuous functions by means of polynomials with fixed degree, Soobsheniya Kharkovskogo Matem. Obshestva, (1912), (in Russian).

[3] N.I. Akhiezer, Lectures in Approximation Theory, 3rd ed., Nauka, Moscow 1972; English transl. Theory of Approximation, Dover Publ., New York 1992.

[4] G. V. Milovanović, D. S. Mitrinović, and Th. M. Rassias, Topics in polynomials: extremal problems, inequalities, zeros, World Scientific Publishing Co., Inc., River Edge, NJ, 1994. MR:1298187(95m:30009)

[5] M. Abramowitz and I. Stegun (Editors), Handbook of Mathematics Functions, National Bureau of Standards, 10-th Edition, NY, 1972.

[6] F. Gantmacher Theory of matrix, v.1, Am. Math. Soc., 2000.

[7] Frank Bowman, Introduction to Bessel functions, Dover Publications Inc., New York, 1958. MR0097539(20 \#4007) 
[8] A. I. Aptekarev, A. Dro, and V. A. Kalyagin, On the asymptotics of exact constants in Markov-Bernstein inequalities in integral metrics with classical weight (Russian), Uspekhi Mat. Nauk 55 (2000), no. 1(331), 173-174, DOI 10.1070/rm2000v055n01ABEH000251; English transl., Russian Math. Surveys 55 (2000), no. 1, 163-165. MR 1751821

[9] André Draux and Charaf Elhami, On the positivity of some bilinear functionals in Sobolev spaces, J. Comput. Appl. Math. 106 (1999), no. 2, 203-243, DOI 10.1016/S03770427(99)00063-1. MR 1696408(2001f:33012)

[10] Alexandre I. Aptekarev, André Draux, and Dmitrii Toulyakov, Discrete spectra of certain co-recursive Pollaczek polynomials and its applications, Comput. Methods Funct. Theory 2 (2002), no. 2, 519-537, DOI 10.1007/BF03321863. MR2038136 (2005c:33006)

[11] André Draux and Valeri Kaliaguine, Markov-Bernstein inequalities for generalized Hermite weight, East J. Approx. 12 (2006), no. 1, 1-23. MR2294666 (2008e:33016)

[12] A. I. Aptekarev, Asymptotics of orthogonal polynomials in a neighborhood of endpoints of the interval of orthogonality (Russian, with Russian summary), Mat. Sb. 183 (1992), no. 5, 43-62, DOI 10.1070/SM1993v076n01ABEH003400; English transl., Russian Acad. Sci. Sb. Math. 76 (1993), no. 1, 35-50. MR1184309 (95c:33006)

[13] D. N. Tulyakov, On the local asymptotics of the ratio of orthogonal polynomials in a neighborhood of an extreme point of the support of the orthogonality measure (Russian, with Russian summary), Mat. Sb. 192 (2001), no. 2, 139-160, DOI 10.1070/SM2001v192n02ABEH000547; English transl., Sb. Math. 192 (2001), no. 1-2, 299-321. MR1835990 (2002d:42029)

[14] D.N. Tulyakov, Difference equations having bases with powerlike growth which are perturbed by a spectral parameter, Matem. sb., 200:5 (2009), 129-158; English transl. in Sb. Math., 200:5 (2009), 753-781.

[15] G. Szegő, Orthogonal polynomials, rev. ed., Amer. Math. Soc, Providence, RI, 1959.

Keldysh Institute of Applied Mathematics, Russian Academy of Sciences, Moscow, Russia and Moscow State University, Moscow, Russia

E-mail address: aptekaa@keldysh.ru

INSA, Rouen, France

E-mail address: andre.draux@insa-rouen.fr

National Research University Higher School of Economics, Nizhny Novgorod, Russia E-mail address: vkalyagin@hse.ru

Keldysh Institute of Applied Mathematics, Russian Academy of Sciences, Moscow, RUSSIA

E-mail address: dntulyakov@gmail.com 